\title{
In Utero Drug Exposure
}

National Cancer Institute

\section{Source}

National Cancer Institute. In Utero Drug Exposure. NCI Thesaurus. Code C98954.

Exposure of the fetus to drugs that are used by the pregnant mother. It may affect fetal growth and result in withdrawal symptoms in the term infant. 